\title{
Optical sensors of bulk refractive index using optical fiber resonators
}

M. Eryürek, Y. Karadag, M. Ghafoor, N. Bavili, K. Cicek, et al.

M. Eryürek, Y. Karadag, M. Ghafoor, N. Bavili, K. Cicek, A. Kiraz, "Optical sensors of bulk refractive index using optical fiber resonators," Proc. SPIE 10231, Optical Sensors 2017, 102312U (16 May 2017); doi:

$10.1117 / 12.2270740$

SPIE. Event: SPIE Optics + Optoelectronics, 2017, Prague, Czech Republic 


\title{
Optical sensors of bulk refractive index using optical fiber resonators
}

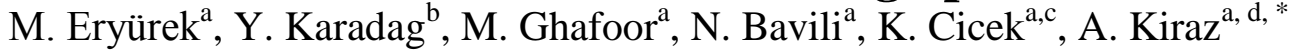 \\ ${ }^{a}$ Department of Physics, Koç University, Rumelifeneri Yolu 34450 Sarıyer, İstanbul, Turkey \\ bístiklal Street, Birlik Avenue No: 23, Ümraniye, İstanbul, Turkey \\ ${ }^{c}$ Department of Electrical and Electronics Engineering, Iğdır University, Suveren Campus, 76000, \\ Iğdır, Turkey \\ ${ }^{\mathrm{d}}$ Department of Electrical and Electronics Engineering, Koç University, Rumelifeneri Yolu 34450 \\ Sarıer, İstanbul, Turkey
}

\begin{abstract}
Optical fiber resonator (OFR) sensor is presented for bulk liquid refractive index (RI) sensing. The sensing mechanism relies on the spectral shifts of whispering gallery modes (WGMs) of OFRs which are excited using a tapered fiber. OFR liquid RI sensor is fully characterized using water solutions of ethanol and ethylene glycol (EG). A good agreement is achieved between the analytical calculations and experimental results for both TE and TM polarizations. The detection limit for bulk RI is calculated to be between $2.7-4.7 \times 10^{-5}$ refractive index unit (RIU). The OFR sensor provides a robust, easy-to-fabricate and sensitive liquid refractive index sensor which can be employed in lab-on-a-chip applications.
\end{abstract}

Keywords: Fiber resonator, WGM, fiber tapering, optical sensor, liquid refractometry, ethanol, ethylene glycol

\section{INTRODUCTION}

Optical whispering gallery modes (WGMs) are very sensitive to changes in the ambient environment ${ }^{1}$. Up to date, this phenomenon inspired many WGM-based sensing studies ${ }^{2}$. Microresonators having high quality factors (Q factors) and considerable evanescent fields extending into the ambient environment have been used with different geometries such as microspheres $^{3}$, microrings ${ }^{4}$, microdisks ${ }^{5}$, liquid-core optofluidic ring resonators ${ }^{6}$, toroids ${ }^{7}$, and optical fiber resonators $(\mathrm{OFRs})^{8}$. Despite the simplicity of OFRs, they did not get much attention for sensing applications except the work reported in Ref. ${ }^{8}$. However, in Ref. ${ }^{8}$, OFRs were investigated only under pure water or pure ethanol environments, and analytical calculations for WGM spectral shifts were not provided.

Here, we use an OFR sensor to detect refractive index (RI) changes of a liquid ambient. Different concentrations of ethanol/water and ethylene glycol (EG)/water solutions are utilized to analytically and experimentally test RI sensing by means of OFR. The WGMs of the OFR are excited from a tunable laser (Santec TSL-510-C, tuning range: $1500-$ $1630 \mathrm{~nm}$, wavelength resolution: $1 \mathrm{pm}$ ) through a tapered fiber which is placed perpendicularly to the $\mathrm{OFR}^{8}$. Then the sensing response of the OFR is observed from the transmission spectrum which reveals the WGMs as dips. In the transmission spectra, both TE and TM modes are observed and analyzed for RI sensing. TM modes possess slightly smaller wavelength than the TE modes for the same mode number. However, TM modes show slightly larger shifts than the TE modes in case of an RI change in the sensing ambient. These observations are also supported and validated by the analytical calculations of the WGMs. The limit of detection (LOD) is calculated to be in the order of $10^{-5}$ refractive index unit (RIU), which is comparable to the state-of-the-art ${ }^{6}$. This LOD can be further improved down to $10^{-7}$ RIU by thermal stabilization control in the ambient environment ${ }^{3,9}$. Depending on the polarization of the WGM, sensitivities are slightly different from each other. From TM and TE mores, average concentration sensitivities for ethanol and EG are demonstrated to be 37 and $67 \mathrm{pm} / \%$, respectively.

*akiraz@ku.edu.tr; phone 90212338 1701; fax 90212338 1559; kirazlab.ku.edu.tr

Optical Sensors 2017, edited by Francesco Baldini, Jiri Homola, Robert A. Lieberman,

Proc. of SPIE Vol. 10231, 102312U · C) 2017 SPIE · CCC code: 0277-786X/17/\$18

doi: $10.1117 / 12.2270740$ 


\section{EXPERIMENTAL}

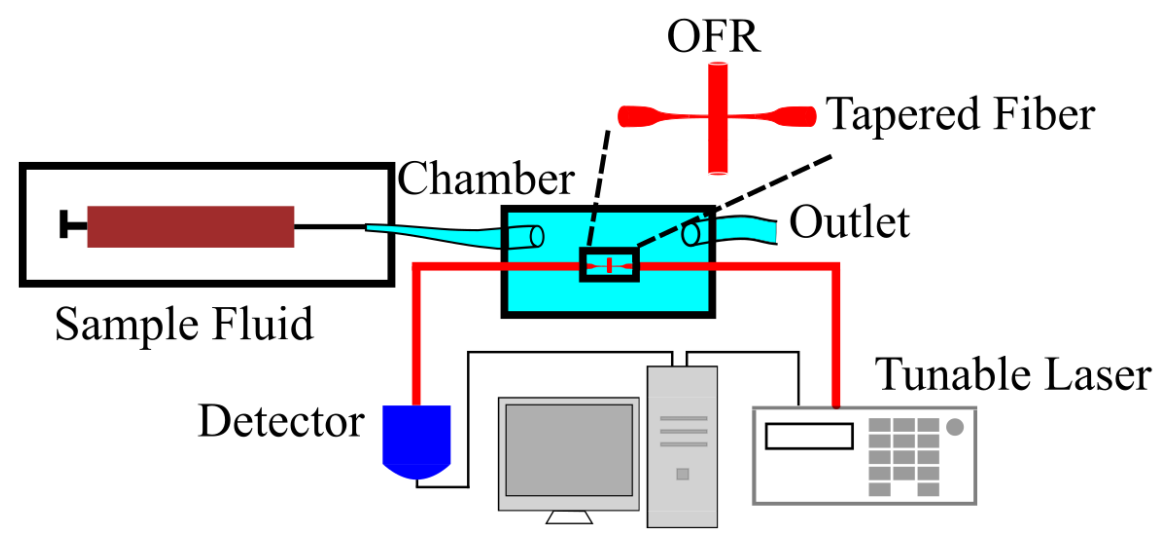

Figure 1. Experimental setup used for measurement of liquid bulk RI. Once the tapered fiber is prepared, OFR is placed perpendicularly on top of the tapered fiber. Then WGMs are excited from the laser and the structure is fixed. Finally, it is placed inside the chamber where the RI of the ambient can be controlled from the syringe pump.

The experimental setup is given in Figure 1. For the experiments, first a single mode fiber (SMF) is tapered for excitation of the WGMs in the OFR. The polymeric coating layer of the SMF is mechanically removed and SMF is wiped clean with isopropyl alcohol (IPA). Then SMF is clamped from both sides onto two independent motorized stages. These stages are pulled in opposite directions while a flame is applied onto the SMF which is generated by burning hydrogen gas flowing with a constant rate of $95 \mathrm{ml}_{n} / \mathrm{min}$. Pulling distance of $\sim 9.2 \mathrm{~mm}$ is applied for each individual motorized stage so that a tapered region of diameter $\sim 3.2 \mu \mathrm{m}$ (See Figure 2) is achieved. This value of the diameter is enough for excitation since the operation wavelength is around $1550 \mathrm{~nm}$. Usually after tapering, the tapered region is not straight and might present a saggy feature. Therefore, an additional pulling of $0.02-0.10 \mathrm{~mm}$ is applied without the flame until the tapered region is straight so that a robust tapered fiber can be obtained.

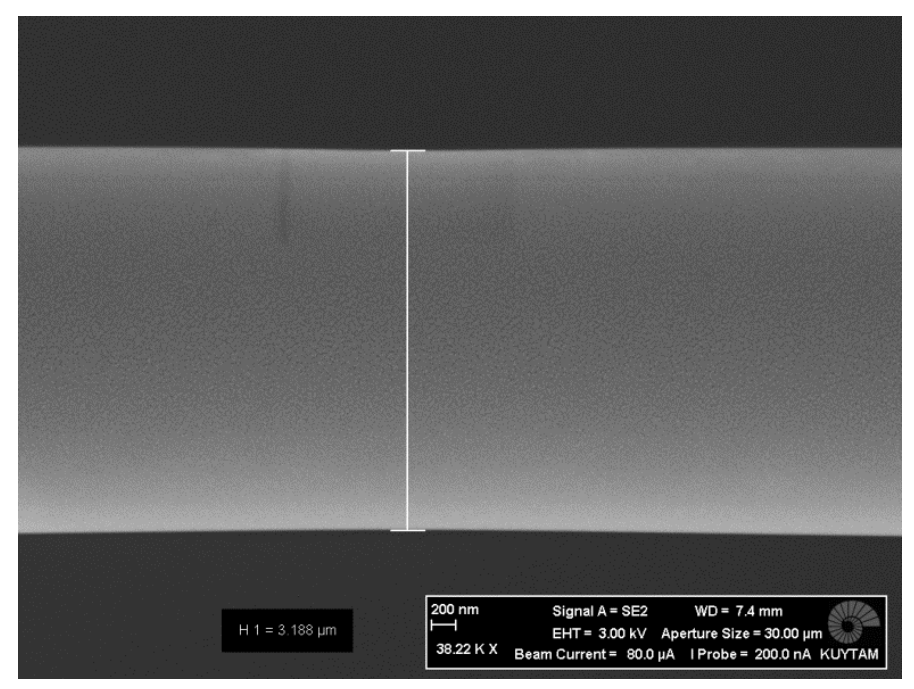

Figure 2. SEM image of the tapered fiber used for the excitation. The diameter of the tapered region is measured to be $\sim 3.2 \mu \mathrm{m}$. This width is small enough to excite the modes of the OFR since the wavelength of the light is around $1550 \mathrm{~nm}$.

After the preparation of tapered fiber, another piece of the same SMF is used as the OFR. Before placing the OFR perpendicular to the tapered region, the polymeric layer is mechanically removed and fiber is cleaned with IPA. Once the WGM dips appear (see Figure 3a and 3b), both the tapered fiber and OFR are fixed from both sides using an epoxy glue 
on a piece of printed circuit board (see Figure 3c). The whole structure is then transported to a liquid chamber where the RI of the ambient environment can be controlled precisely by injecting water solutions of ethanol or EG through a syringe pump (see Figure 3d). In the air, a Q factor of 63000 (Figure 3a) is measured, but this value decreases down to 16000 (Figure 3b) when the ambient environment switches to water. This change is attributed to the absorption and scattering losses induced by the contaminants in the liquid and absorption loss induced by the water.
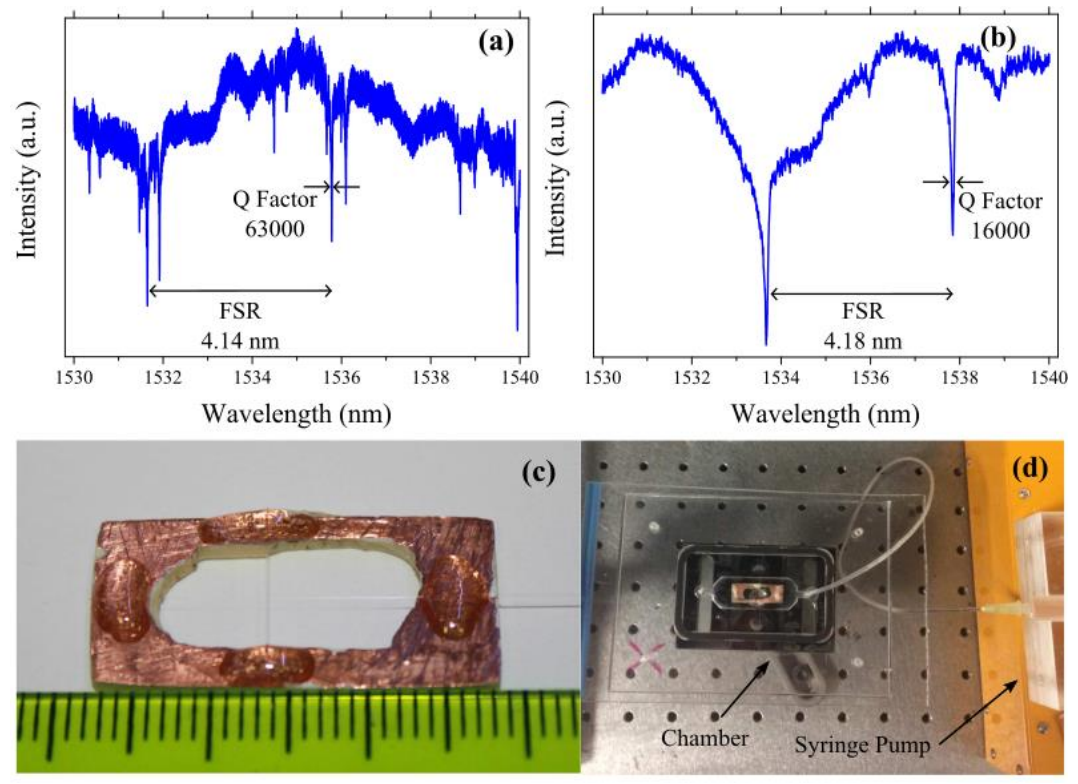

Figure 3. Transmission spectra when the OFR is in a) air ambient and b) water ambient. c) Close view of the OFR sensor and the tapered fiber. Both the tapered fiber and OFR are fixed using an epoxy glue on a piece of printed circuit board. Each small division given in the ruler corresponds to $1 \mathrm{~mm}$. d) Top view of the bulk liquid RI sensing experimental setup. After the OFR sensor is transferred to the chamber, the RI of the ambient environment is controlled by injecting water solutions of ethanol or EG at required concentrations.

\section{ANALYTICAL CALCULATIONS}

For cylindrical resonators, WGMs having TM and TE polarizations appear when their respective field amplitudes $a_{l}$ and $b_{l}$ given below tend to infinity ${ }^{10}$.

$$
\begin{aligned}
a_{l} & =\frac{J_{l}^{\prime}(y) J_{l}(x)-m J_{l}(y) J_{l}^{\prime}(x)}{J_{l}^{\prime}(y) H_{l}(x)-m J_{l}(y) H_{l}^{\prime}(x)} \\
b_{l} & =\frac{m J_{l}^{\prime}(y) J_{l}(x)-J_{l}(y) J_{l}^{\prime}(x)}{m J_{l}^{\prime}(y) H_{l}(x)-J_{l}(y) H_{l}^{\prime}(x)}
\end{aligned}
$$

where $J_{l}$ is the $l^{t h}$-order Bessel function of the first kind, $H_{l}$ is the $l^{t h}$-order Hankel function of the first kind and $m$ is the relative RI between the ambient and the resonator, i.e. $m=n_{\text {in }} / n_{\text {out }}$. The arguments of the Bessel and Hankel functions are defined as $x=2 \pi R n_{\text {out }} / \lambda$, also known as the size parameter and $y=m x . R, \lambda$ and $l$ represent the radius of the resonator, vacuum wavelength of the light and azimuthal mode number, respectively. Using the values $n_{\text {out }}=1$, $R=62.5 \mu \mathrm{m}$ and $\lambda=1535 \mathrm{~nm}$, the free spectral ranges (FSRs) for both TM and TE polarizations using Equations 1 and 2 are calculated as $4.15 \mathrm{~nm}$ and $4.16 \mathrm{~nm}$ respectively when $n_{i n}=1.46$ is used to fit the observed FSR values in the air and in the water $\left(4.14 \mathrm{~nm}\right.$ and $4.18 \mathrm{~nm}$ respectively. See Figures $3 \mathrm{a}$ and $3 \mathrm{~b}$ ). Value for $n_{\text {in }}$ used in the FSR calculation is in agreement with the typical glass materials for $\lambda=1535 \mathrm{~nm}^{11}$. With the given parameters and $l=362$, the WGMs are calculated to be at $1537.68 \mathrm{~nm}$ and $1536.17 \mathrm{~nm}$ for TE and TM polarizations, respectively. 


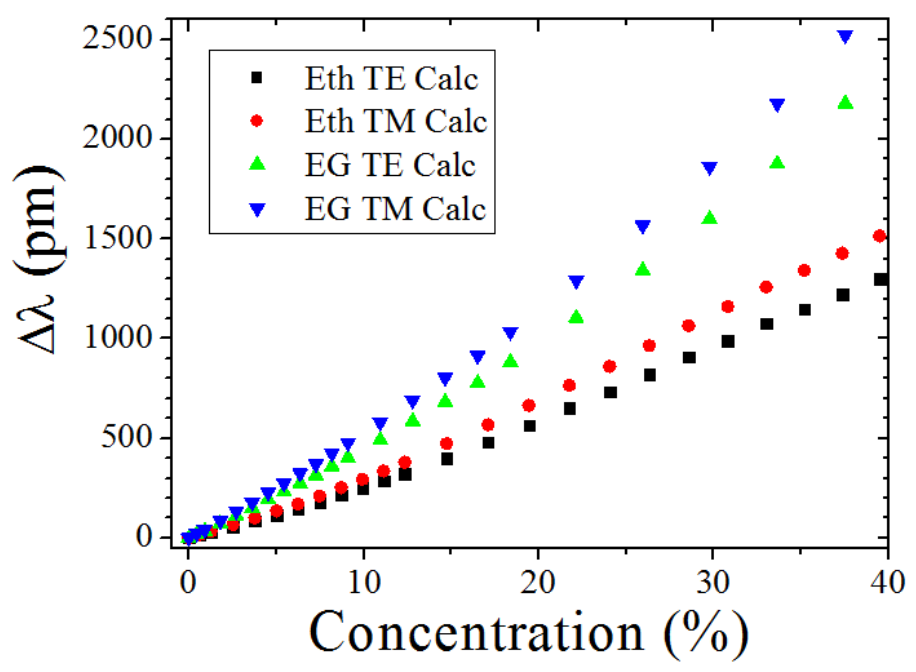

Figure 4. Calculated spectral shift values as a function of ethanol or EG concentration, both given for TM and TE polarizations. EG is calculated to cause more spectral shift per unit concentration change because RI of water/EG solution increases more than the RI of ethanol/water solution.

The analytical calculation of spectral shift with respect to concentration of ethanol $\left(c_{E T H}\right)$ or EG $\left(c_{E G}\right)$ is performed utilizing the RI values of ethanol and EG solutions at $\lambda=589 \mathrm{~nm}$ provided in reference ${ }^{12}$. These values are corrected to RI values at $\lambda=1535 \mathrm{~nm}$ using normalization factors obtained from the information given $\mathrm{in}^{11}$. Water, ethanol and EG RI values are taken to be 1.3183, 1.3506 and 1.4199, respectively at $\lambda=1535 \mathrm{~nm}$. Using Equations 1 and 2 and the RI values obtained from references ${ }^{11,12}$, the expected spectral shifts of WGMs are calculated for both TM and TE polarizations and depicted in Figure 4. For small ethanol or EG concentration changes around 0\%, bulk RI sensitivities are calculated as $\sim 63 \mathrm{~nm} / R I U$ and $\sim 55 \mathrm{~nm} / R I U$ for TM and TE polarizations, respectively. Meanwhile, ethanol and EG sensitivities are calculated to be 26 (23) $\mathrm{pm} / \%$ and 51 (46) $\mathrm{pm} / \%$ for TM (TE) polarization, respectively.
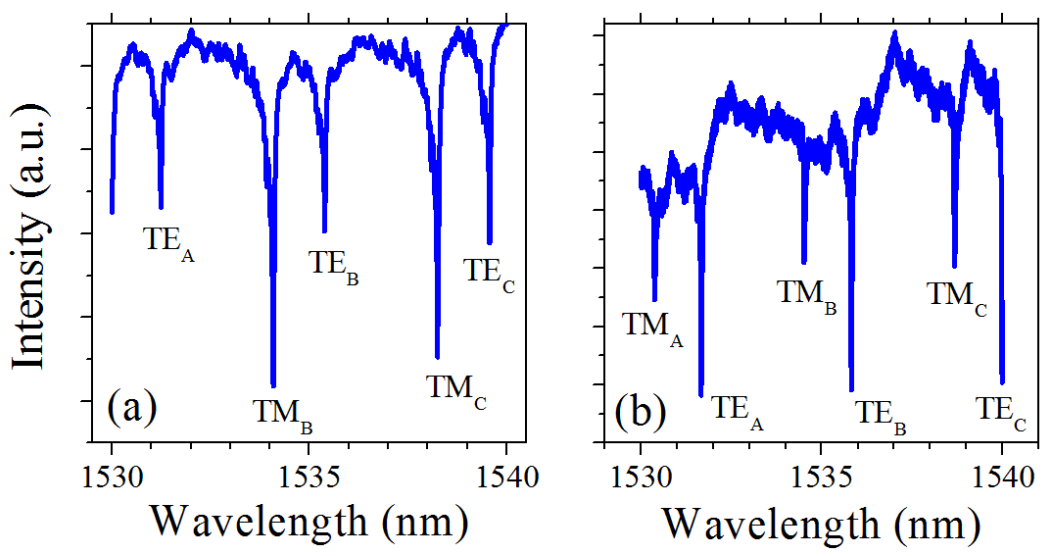

Figure 5. Transmission spectra recorded in the beginning of a) ethanol and b) EG experiments. Both TE and TM mode families can be observed. According to the analytical calculations, for a given mode number, TM modes have smaller wavelength than TE modes. Modes having the same mode number are designated with the same subscript; A, B or C. Slight shift at the mode positions between the modes in (a) and (b) is attributed to slight change in the effective radius of the WGMs as a result of slight deviation of the angle between the OFR and the tapered fiber.

In the transmission spectra, both TM and TE mode families are observed as depicted in Figure 5. In each measurement, one WGM is selected and analyzed using a Gaussian fitting algorithm ${ }^{13}$ under changing RI of the ambient environment. Smaller wavelength for the same mode number is considered as the TM mode based on the result from the analytical 
calculations. Subscripts A, B and C represent different azimuthal mode numbers for TM or TE polarized modes depicted in Figure 5. Slight deviation of the spectral positions between Figure 5a and Figure 5b is attributed to different effective radii of the OFR as a result of the slight deviation of the angle between the tapered fiber and the OFR for different experiments.

\section{EXPERIMENTAL RESULTS}

Exemplary time traces of ethanol and EG measurements are depicted in Figure 6. Staircase (a and b) and hysteresis (c and d) experiments reveal that resonance shifts closely follow the changes in the RI of the medium. Staircase experiments show that OFR can perform under low or high concentrations of ethanol/EG, whereas hysteresis experiments indicate the OFR is stable for long durations of time. In addition, the zero level is conserved and response and recovery times are in the order of minutes. Hysteresis is defined as the difference between $\Delta \lambda_{\text {forw }}$ and $\Delta \lambda_{\text {back }}$, normalized to $\Delta \lambda_{\text {forw }}$, where $\Delta \lambda_{\text {forw }}$ and $\Delta \lambda_{\text {back }}$ are the spectral shift values during the increase and decrease of solute concentration. From Figure $6 \mathrm{c}$ and Figure $6 \mathrm{~d}$, hysteresis is calculated to be $15 \%$ and $2 \%$ for ethanol and EG, respectively.
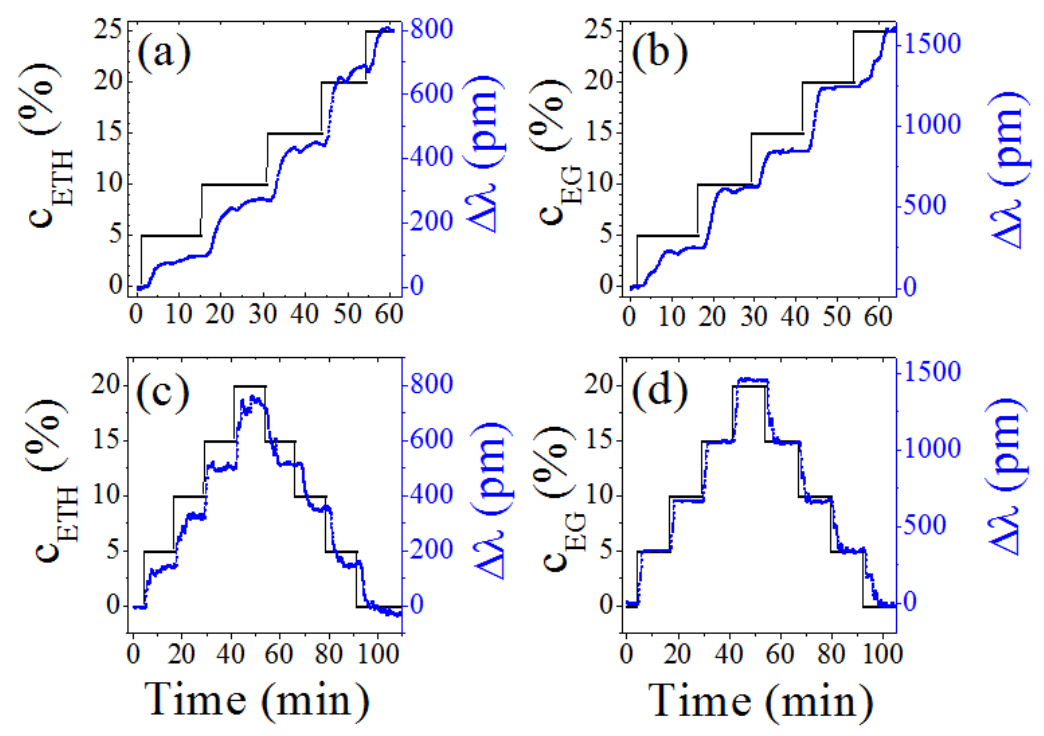

Figure 6. Time traces of a)-c) ethanol and b)-d) EG experiments. Two different kinds of experiments performed which are a)-b) staircase and c)-d) hysteresis. Fluctuations in the resonance shift are attributed to adding of multiple injections of the solution until the RI in the ambient reaches to steady state. Response time and recovery time are in the order of minutes and zero level is conserved. Hysteresis of $17 \%$ and $2 \%$ are calculated from (c) and (d).

In Figure 7, comparison between the analytical calculations and experimental results is depicted. Experimental results and analytical calculations agree with each other, proving the reliability of the OFR sensor. In the concentration range of $c_{E T H}=0-30 \%$, average ethanol concentration sensitivities are $39(\mathrm{TM})$ and $34 \mathrm{pm} / \%$ (TE) as compared to the calculated sensitivities of 37.7 (TM) and $32.1 \mathrm{pm} / \%$ (TE). For EG, between $c_{E G}=0-30 \%, 71$ (TM) and $63 \mathrm{pm} / \%$ sensitivities are obtained experimentally whereas the calculated sensitivities are 62.5 (TM) and $53.8 \mathrm{pm} / \%$ (TE). Concentration sensitivity of EG is higher because the RI change of EG solution per concentration is more than ethanol solution. Mode families having TM and TE polarizations have different sensitivities, therefore these two mode families split in the sensitivity plots (see Figure $7 \mathrm{a}$ and $7 \mathrm{~b}$ ).

Standard deviation in the wavelength measurement is calculated to investigate the LOD of the OFR. From 75 data points in Figure $6 \mathrm{c}$ between $t=59.56$ and 68.68 minutes, the standard deviation in wavelength measurement for ethanol is calculated as $2.6 \mathrm{pm}$. For EG, in Figure $6 \mathrm{~d}$, between $t=6.75$ and 16.65 minutes and from 80 data points, standard deviation is calculated as $1.7 \mathrm{pm}$. Considering these values as the minimum detectable spectral shifts, LOD of ethanol and EG are calculated to be $4.1 \times 10^{-5}\left(4.7 \times 10^{-5}\right)$ and $2.7 \times 10^{-5}\left(3.0 \times 10^{-5}\right)$ RIU for TM (TE) polarization. 

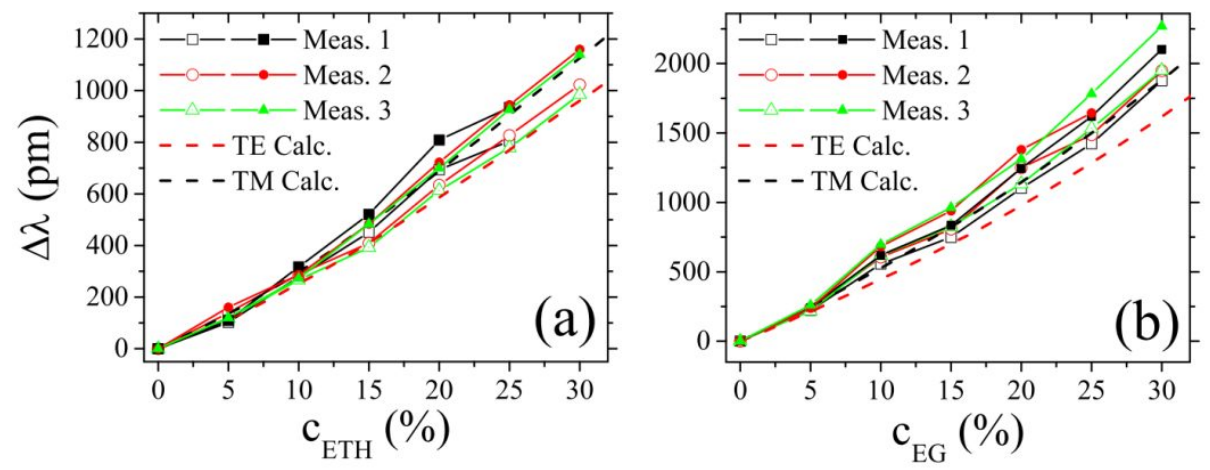

Figure 7. Comparison between the analytical calculations and the experimental results for a) ethanol and b) EG. Three measurements performed both for ethanol and EG show good agreement with the analytical calculations depicted as dashed lines. In addition, a split can be observed between different mode families which have different polarizations. Modes having TM polarization are designated with full shapes, whereas TE modes are designated with void shapes. Each different shape (square, disc or triangle) correspond to different measurements.

\section{CONCLUSION}

In this work, an optical fiber resonator (OFR) is employed in sensitive detection of bulk liquid RI sensing, and analyzed both experimentally and analytically. Sensing mechanism takes advantage of the spectral shifts of WGMs excited in the OFR using a tapered fiber. Liquid RI sensing is investigated using ethanol or EG solutions of water. Concentration sensitivities of ethanol and EG are obtained as 39 (34) and 71 (63) $\mathrm{pm} / \%$ respectively for TM (TE) polarizations in the concentration range of $0-30 \%$. These values are also verified with analytical calculations. LOD of the system is between $2.7 \times 10^{-5}$ and $4.7 \times 10^{-5}$ RIU, which can be further improved using thermal control of the ambient environment. OFR sensor used in bulk liquid RI detection has a high potential to be used in lab-on-a-chip and biological sensing applications due to its ease of fabrication, reliability, compactness and high sensitivity.

\section{REFERENCES}

[1] Vahala, K. J., “Optical microcavities”, Nature 424, 839-846 (2003).

[2] Foreman, M. R., Swaim, J. D. and Vollmer, F., "Whispering gallery mode sensors", Advances in Optics and Photonics 7, 168-240 (2015).

[3] Hanumegowda, N. M., Stica, C. J., Patel, B. C., White, I. M. and Fan, X., "Refractometric sensors based on microsphere resonators", Applied Physics Letters 87 (20), 201107 (2005).

[4] Levy, U., Campbell, K., Groisman, A., Mookherjea, S. and Fainman, Y., "On-chip microfluidic tuning of an optical microring resonator", Applied Physics Letters 88, 111107 (2006)

[5] Eryürek, M., Tasdemir, Z., Karadag, Y., Anand, S., Kilinc, N., Alaca, B. E. and Kiraz, A., "Integrated humidity sensor based on SU-8 polymer microdisk microresonator", Sensors and Actuators B: Chemical 242, 1115-1120 (2017).

[6] White, I. M., Oveys, H. and Fan X., "Liquid-core optical ring-resonator sensors", Optics Letters 31 (9), 1319-1321 (2006).

[7] Armani, A. M. and Vahala, K. J., "Heavy water detection using ultra-high-Q microcavities", Optics Letters 31 (12), 1896-1898 (2006).

[8] Boleininger A., Lake, T., Hami, S. and Vallance C., "Whispering gallery modes in standard optical fibres for fibre profiling measurements and sensing of unlabelled chemical species", Sensors 10, 1765-1781 (2010).

[9] Li, H. and Fan, X., "Characterization of sensing capability of optofluidic ring resonator biosensors", Applied Physics Letters, 97 (1), 011105 (2010)

[10] van de Hulst, H. C., [Light Scattering by Small Particles ], Dover Publications Inc., New York, 297-301 (1981). 
[11] Polanskiy, M. N., "Refractive index database", http://refractiveindex.info (16 March 2017).

[12]Lide, D. R., [CRC Handbook of Chemistry and Physics], CRC Press, Internet Version 8-63 - 8-64 (2005).

[13] Eryürek, M., Karadag, Tasaltin, N., Y., Kilinc, N. and Kiraz, A., "Optical sensor for hydrogen gas based on a palladium-coated polymer microresonator", Sensors and Actuators B: Chemical 212, 78-83 (2015). 